\title{
Beam Tilt-Angle Estimation for Monopole End-Fire Array Mounted on a Finite Ground Plane
}

\author{
Jia Cao, Zhenghui Xue, and Meng Cao \\ School of Information and Electronics, Beijing Institute of Technology, 5 South Zhongguancun Street, Haidian District, \\ Beijing 100081, China \\ Correspondence should be addressed to Zhenghui Xue; zhxue@bit.edu.cn
}

Received 2 October 2014; Revised 2 December 2014; Accepted 15 December 2014

Academic Editor: Ana Alejos

Copyright (C) 2015 Jia Cao et al. This is an open access article distributed under the Creative Commons Attribution License, which permits unrestricted use, distribution, and reproduction in any medium, provided the original work is properly cited.

\begin{abstract}
A modified method for the beam tilt-angle estimation of monopole end-fire array mounted on finite ground plane is proposed. In the simplified model, the monopole array and ground plane are approximated to two line sources of transverse and longitudinal electric current, respectively. It is deduced that the beam tilt angle is a function about the length of ground plane in front of array $L_{g}$, the length of monopole array $L_{a}$, and the phase constant $\beta_{\alpha}$. After verifying the optimizing principle of monopole end-fire array, a 10-element monopole Yagi-Uda antenna satisfying Hansen-Woodyard condition is designed and measured for the analysis. By comparison and analysis, the value of $\beta_{\alpha}$ is demonstrated to be the key point of the proposed method. And a slow wave monopole array is proved to be able to achieve a low beam tilt angle from end-fire with only a short-length ground plane.
\end{abstract}

\section{Introduction}

End-fire antennas are useful in all applications that require low profile, for example, in airborne and vehicular environments. After decades of research and developing, the performance of end-fire antennas has been greatly advanced in several fields, like broadband, gain enhancement, and pattern reconfiguration [1-4]. However, those end-fire antennas mounted in or on a finite ground plane suffer from a serious problem: the main beam is tilted at some angle from the horizontal plane by diffraction effects from conductive ground, which prevents a true end-fire condition.

By image theory, the conclusion can be easily obtained that the beam tilt angle will disappear when ground plane extends to infinite. Meanwhile, as a majority of radiation power distributes in the positive half-space, the value of the beam tilt angle mainly depends on the effective ground plane length in front of end-fire antenna. This phenomenon was first described by Elliott in 1954 [5].

Based on Huygens' principle, a simplified method for determining the effect of finite ground plane was demonstrated by Walter in his book, by which the aperture of antenna and the ground plane were simplified to a uniform line source of magnetic current and electric current, respectively. As a result, the beam tilt $\theta_{t}$ was proved to be a function of both $L_{a}$ (given antenna length) and $L_{g}$ (effective ground plane length). For small values of beam tilt $\left(\theta_{t} \leq 20^{\circ}\right)$, this function reduced to

$$
\theta_{t} \approx \frac{49}{\left[\left(L_{a}+L_{g}\right) / \lambda\right]^{1 / 2}}
$$

where $\theta_{t}$ is in degrees [6].

Nevertheless, this method with high accuracy is not suitable for the monopole end-fire array vertical to the ground plane. That is not only because of the model abstracted from the flush-mounted end-fire antenna which is a kind of aperture antenna parallel to the ground, but also due to no consideration about the influence from phase constant's varying. Thus in the former research, the beam tilt-angle analysis of this kind of monopole end-fire array could only be obtained by whole model simulation using simulation software, far-field measurement, or numerical calculation method [7], with high cost, long time, and various operations.

A beam tilt-angle estimation method, which is modified for the monopole end-fire array mentioned above, is 


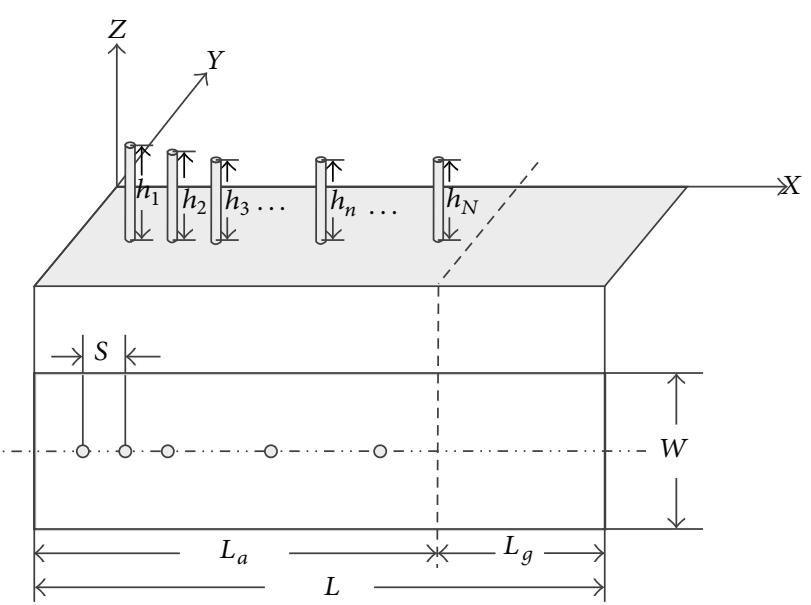

FIGURE 1: $N$-monopole end-fire antenna vertically mounted on a finite ground plane.

proposed in this paper. Firstly, a simplified source model is applied to this kind of antenna. The key point of this model simplification is evaluating the phase constant $\beta_{a}$ of the surface wave along monopole end-fire array. Secondly, monopole Yagi-Uda antenna is introduced to validate the validity and accuracy of this method. The estimated tilt angles present a good agreement with CST simulation results. Furthermore, comparison between the proposed method in this paper and Walter's method shows an advantage of monopole end-fire antenna with slow wave characteristic. This advantage, that a low tilt angle can be achieved with a short ground length, is demonstrated by the experimental results of the 10-element monopole Yagi-Uda antenna.

\section{Simplified Model for Monopole End-Fire Array and Ground Plane}

Figure 1 shows the configuration of an $\mathrm{N}$-monopole endfire array mounted on a ground plane with certain size $(W L)$. Assume it is working at the frequency point $f$, with a wavelength $\lambda$, and $\omega$ is the corresponding angular frequency. Consulting the original method in [6], the simplified model should be composed of two parts: one line source approximated from the monopole array current distribution and another one approximated from the current on the ground plane. Note that only far-field patterns in $X Z$-plane are taken into account for the beam tilt-angle problem, as the main lobe direction is always in this plane when the ground is symmetric about the $X$-axis.

2.1. Simplification for Monopole End-Fire Array. Firstly, the array elements, electric monopoles with heights $\left\{h_{n}\right\}$, are approximated by a series of ideal dipoles. Ideal dipole is defined as a Hertz dipole with uniform electric current $I$ on the total length $\Delta l \ll \lambda$, and its radiation pattern function is $f=\sin \theta$. Usually, monopole height is less than or equal to $0.25 \lambda$. Assuming the current distribution on monopole is $I(z)=I_{m} \sin (k(h-z))$, where $h$ is the

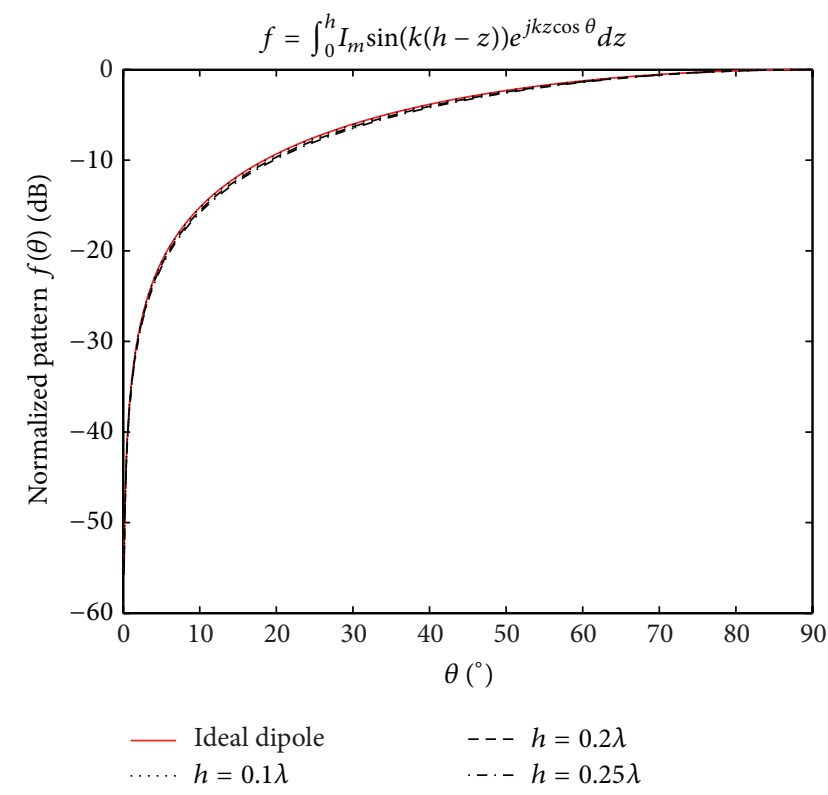

FIGURE 2: Radiation patterns of $0.1 \lambda, 0.2 \lambda$, and $0.25 \lambda$ monopoles and of ideal dipole.

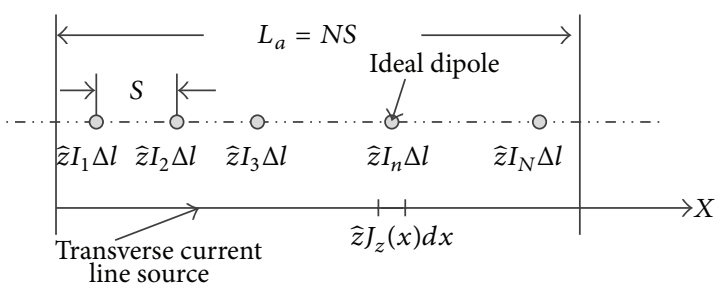

Figure 3: Ideal dipole array and transverse current line source corresponding.

height of monopole, the radiation pattern can be derived by (2). Figure 2 exhibits the curves of $E$-plane ( $X Z$-plane) normalized patterns of ideal dipole, $0.1 \lambda$ monopole, $0.2 \lambda$ monopole, and $0.25 \lambda$ monopole, respectively. It is suggested that ideal dipole's radiation pattern is quite similar to the ones of monopoles with height less than or equal to $0.25 \lambda$, so this approximation has no serious loss in accuracy:

$$
f=\int_{0}^{h} I_{m} \sin (k(h-z)) e^{j k z \cos \theta} d z
$$

Secondly, as shown in Figure 3, the array of ideal dipoles with electric current amplitude and phase offset as $\left(A_{n}, \varphi_{n}\right)$ on each is approximated by a transverse electric current line source lying on the $X$-axis, with amplitude $A(x)$ and phase velocity $v(x)$ in $X$ direction. For the line source length, assume that each ideal dipole $\widehat{z} I_{n} \Delta l=\widehat{z} A_{n} e^{j \varphi_{n}}$ lying on $x_{n}$ corresponds to a segment $s$ (spacing between two adjacent elements) in length, so the whole length of line source will be

$$
L_{a}=N s,
$$


where $N$ is the number of elements. Therefore, let the current source be

$$
J_{z}(x)=A(x) e^{-j \beta(x) x} \quad 0 \leq x \leq L_{a},
$$

where

$$
\beta(x)=\frac{\omega}{v(x)}
$$

is the phase constant on the line source. For small element spacing (one-half wavelength or less) the distinction between array and continuous source tends to disappear. So when $s \leq$ $0.5 \lambda$, this approximation works.

Finally, simplify the line source expressed by (3) to a line source with a constant amplitude $A_{a}$ and uniform phase constant $\beta_{a}$. For beam-tilting estimation, only a relative value of $A_{a}$ is needed, and this work will be introduced in Section 2.3. Meanwhile the value of $\beta_{a}$ is evaluated as

$$
\beta_{a}=\frac{\sum_{n=2}^{N}\left(\varphi_{n}-\varphi_{n-1}\right)}{L_{a}} .
$$

$\beta_{a}$ is just the key point of this new method, owing to the fact that the difference between the value of $\beta_{a}$ and the wavenumber in free space $k$ cannot be ignored in many cases. The further explanation will be given in Section 3 .

According to the simplification above, the line source expression is deduced to

$$
J_{z}(x)=A_{a} e^{-j \beta_{a} x} \quad 0 \leq x \leq L_{a}
$$

whose relative far-field in elevation plane ( $X Z$-plane) is

$$
\widehat{\theta} E_{a}=\widehat{\theta} A_{a} e^{j X_{a}} \frac{\sin X_{a}}{X_{a}} L_{a} \sin \theta \text {, }
$$

where $X_{a}=\left(L_{a} / 2\right)\left(k \sin \theta-\beta_{a}\right)$.

2.2. Simplification for Ground Plane. In electromagnetic field analysis, a ground plane can be replaced by a current sheet with its induced current components $\left(J_{x}, J_{y}\right)$ distributed on. Based on the mirror symmetry of end-fire antenna's structure in Figure 1, $J_{y}$ on the current sheet has no contribution to the far-field in $X Z$-plane, so it can be ignored in the beam tilting estimation. Thus the simplified model for the ground plane should be a line source of longitudinal electric current on $X$ axis.

This line source is divided into two parts: the part representing the ground plane just under the monopoles (G1) and the part representing the ground plane in front of endfire array (G2). The current amplitudes are assumed to be $A_{g}$, equal and constant on both parts. However, the phase constant $\beta(x)$ should not be uniform along the whole line source. The current in G2 is close to the current induced by monopoles' far-field, so the phase constant in G2 is close to $k$. Meanwhile, the current phase constant in G1 approaches to $\beta_{a}$ because of the constraint by monopole array's nearfield but regresses to $k$ at the end of array because of the continuity of $\beta(x)$ on the boundary between G1 and G2.
According to the considerations about both simplicity and accuracy, the phase constant in G1 is valued by the average of $\beta_{a}$ and $k$. The estimation for the phase constant in G1 is another special progressing in the novel method, because in previous work only the ground in front of the end-fire aperture, corresponding to G2 in our case, is taken into account $[5,6]$.

Thus, the simplified line source can be expressed as

$$
\begin{aligned}
& J_{x} \\
& =\left\{\begin{array}{cl}
A_{g} e^{-j\left(\left(\beta_{a}+k\right) / 2\right) x} & 0 \leq x \leq L_{a} \\
A_{g} e^{-j\left[k\left(x-L_{a}\right)+\left(\left(\beta_{a}+k\right) / 2\right) L_{a}\right]} & \\
=A_{g} e^{-j\left(k x+\left(\left(\beta_{a}-k\right) / 2\right) L_{a}\right)} & L_{a}<x \leq L_{a}+L_{g} .
\end{array}\right.
\end{aligned}
$$

And its relative far-field is

$$
\widehat{\theta} E_{g}=\widehat{\theta} A_{g}\left(e^{j X_{g 1}} \frac{\sin X_{g 1}}{X_{g 1}} L_{a}+e^{j X_{g 3}} \frac{\sin X_{g 2}}{X_{g 2}} L_{g}\right) \cos \theta,
$$

where

$$
\begin{aligned}
& X_{g 1}=\frac{L_{a}}{2}\left(k \sin \theta-\frac{\beta_{a}+k}{2}\right), \\
& X_{g 2}=\frac{L_{g}}{2} k(\sin \theta-1), \\
& X_{g 3}=\left(L_{a}+\frac{L_{g}}{2}\right) k(\sin \theta-1)+L_{a} \frac{k-\beta_{a}}{2} .
\end{aligned}
$$

2.3. Superposition of Far-Field Patterns. The total estimated radiation is the superposition of the far-fields of the two line sources introduced in Sections 2.1 and 2.2. Good results are obtained in practice by letting the sources be in-phase at the origin and with amplitudes adjusted so that their pattern maxima are equal by Walter's method [6]. Therefore the same assumption is made in this paper.

Field $E_{a}$ will have maximum value at $\theta=90^{\circ}$. Setting $\left|E_{a}\right|_{\max }=\left|E_{g}\right|_{\max }$ gives

$$
\begin{aligned}
& \left.A_{a} L_{a} \frac{\sin X_{a}}{X_{a}}\right|_{\theta=90^{\circ}} \\
& \quad=A_{g}\left|\left(e^{j X_{g 1}} \frac{\sin X_{g 1}}{X_{g 1}} L_{a}+e^{j X_{g 3}} \frac{\sin X_{g 2}}{X_{g 2}} L_{g}\right) \cos \theta\right|_{\max } .
\end{aligned}
$$


Thus the total far-field is

$$
\begin{aligned}
E_{t}= & E_{a}+E_{g} \\
= & A_{g}\left|\left(e^{j X_{g 1}} \frac{\sin X_{g 1}}{X_{g 1}} L_{a}+e^{j X_{g 3}} \frac{\sin X_{g 2}}{X_{g 2}} L_{g}\right) \cos \theta\right|_{\max } \\
& \times\left.\frac{X_{a}}{\sin X_{a}}\right|_{\theta=90^{\circ}} e^{j X_{a}} \frac{\sin X_{a}}{X_{a}} \sin \theta \\
& +A_{g}\left(e^{j X_{g 1}} \frac{\sin X_{g 1}}{X_{g 1}} L_{a}+e^{j X_{g 3}} \frac{\sin X_{g 2}}{X_{g 2}} L_{g}\right) \cos \theta .
\end{aligned}
$$

The main lobe direction $\theta_{m}$ can be obtained by solving the equation $\left.\left(\partial E_{t} / \partial \theta\right)\right|_{\theta=\theta_{t}}=0$, and the beam tilt angle $\theta_{t}$ has $\theta_{t}=90^{\circ}-\theta_{m}$. So, $\theta_{m}$ is proved to be a function of $L_{a}, L_{g}$, and $\beta_{a}$. Moreover, for the convenience of calculation, numerical computing for (12) to find the value of $\theta$ corresponding to $\left|E_{t}\right|_{\max }$ is also a considerable solution.

\section{Simulation Analysis and Discussion}

A monopole Yagi-Uda antenna is taken as an example of monopole end-fire array for the beam tilting analysis and discussion. In most conditions, conventional Yagi-Uda antenna is inherently a slow wave structure, and Hansen-Woodyard condition which is expressed by (14) is usually considered as the optimization aim in this kind of antenna's design for improving directivity [6]. Before antenna designing, a simulation experiment was implemented to verify that this optimizing principle applies to the monopole end-fire array case. Consider

$$
\beta_{a} L_{a}=\pi+k L_{a}
$$

3.1. Optimizing Principle in Monopole End-Fire Array. The simulation object is a 10 -element monopole array like the model shown in Figure 1, working at $f=3 \mathrm{GHz}$. All the monopoles in this array have an equal height $h=0.25 \lambda=$ $25 \mathrm{~mm}$ and an equal radius $r=2 \mathrm{~mm}$. The interspace between adjacent elements is $s=20 \mathrm{~mm}$, so the total length of array is $L_{a}=200 \mathrm{~mm}$. The finite ground size is $L_{a}=200 \mathrm{~mm}$, $L_{g}=30 \mathrm{~mm}$, and $W=60 \mathrm{~mm}$. To satisfy Hansen-Woodyard condition, the actual phase constant on the array's current should be $\beta_{a}=1.25 \mathrm{k}$.

Every element was fed with an equal magnitude and a uniform-step phase offset. By CST MWS simulating, the fed signal phase step $\Delta \varphi$ between adjacent elements was taken as a variable, the array's directivities and tilt angles with different $\Delta \varphi$ were recorded, and the surface current data of monopoles were exported for calculating the phase constant $\beta_{a}$ on this array.

Specifically, Figure 4(a) shows the surface current distribution on monopoles with a certain phase step $\Delta \varphi_{0}$. By means of processing these current data in Matlab, the actual phase offset of current source on each element $\varphi_{n}$ was calculated and Figure 4(b) shows the results. Substituting $\left\{\varphi_{n}\right\}$ in (6), the actual phase constant $\beta_{a}$ was derived.
TABLE 1: Parameter for monopole Yagi-Uda antenna.

\begin{tabular}{lc}
\hline Variables & Values \\
\hline$r(\mathrm{~mm})$ & 2 \\
$s(\mathrm{~mm})$ & 20 \\
$h 1(\mathrm{~mm})$ & 23 \\
$h 2(\mathrm{~mm})$ & 21.5 \\
$h 3(\mathrm{~mm})$ & 19 \\
$h 4(\mathrm{~mm})$ & 17 \\
$h 5(\mathrm{~mm})$ & 15.6 \\
$h 6(\mathrm{~mm})$ & 15.5 \\
$h 7(\mathrm{~mm})$ & 14.5 \\
$h 8(\mathrm{~mm})$ & 10.67 \\
$h 9(\mathrm{~mm})$ & 6.83 \\
$h 10(\mathrm{~mm})$ & 3 \\
\hline
\end{tabular}

Thus, through this simulation experiment, the relationships between $\beta_{a}$ and array's directivity and $\beta_{a}$ and array's tilt angle were both obtained. The black solid curve in Figure 5 illustrates that the directivity $D \approx 11.43 \mathrm{~dB}$ when $\beta_{a}=1.25 \mathrm{k}$, and it is almost the optimum directivity for this 10-element array. It demonstrates Hansen-Woodyard condition is still suitable for the monopole end-fire array case.

To be worthy of attention, the red solid curve in Figure 5 reveals the trend that beam tilt angle is decreasing while $\beta_{a}$ is increasing.

3.2. Beam Tilting Estimation to a Monopole Yagi-Uda Antenna. A 10-element dipole Yagi-Uda antenna has been designed following Hansen-Woodyard condition which has been verified above at $f=3 \mathrm{GHz}$. And the corresponding monopoles in the positive- $Z$-space are mounted on a finite ground plane to constitute a monopole Yagi-Uda antenna. The configuration is shown in Figure 6, and parameters of the monopole array are given in Table 1.

According to (3), the monopole array's length is $L_{a}=$ $200 \mathrm{~mm}$. Substituting it in (14), the solution is $\beta_{a} \approx 78.54$, whose distinction with $k=2 \pi / \lambda \approx 62.83$ cannot be ignored. Thus only the length of ground plane in front of $L_{g}$ is the variable in this analysis. A $\theta_{t}-L_{g}$ curve is simply calculated by the estimation method proposed in Section 2 . Meanwhile, there are two other $\theta_{t}-L_{g}$ curves calculated by Walter's method and simulated by CST Microwave Studio, respectively. All the three curves are shown in Figure 7 for comparison. The estimated result by Walter's method has a clear distinction to the simulation one. Meanwhile a good agreement is achieved between the modified estimation and CST simulation. The accuracy of the proposed estimation method is illustrated to be much better than Walter's for beam tilt angle of monopole end-fire array like this monopole YagiUda antenna.

Furthermore, the estimated value of this new method is in general lower than Walter's when the finite ground plane is short, and two curves trend to approximation and the length of ground plane increases. 


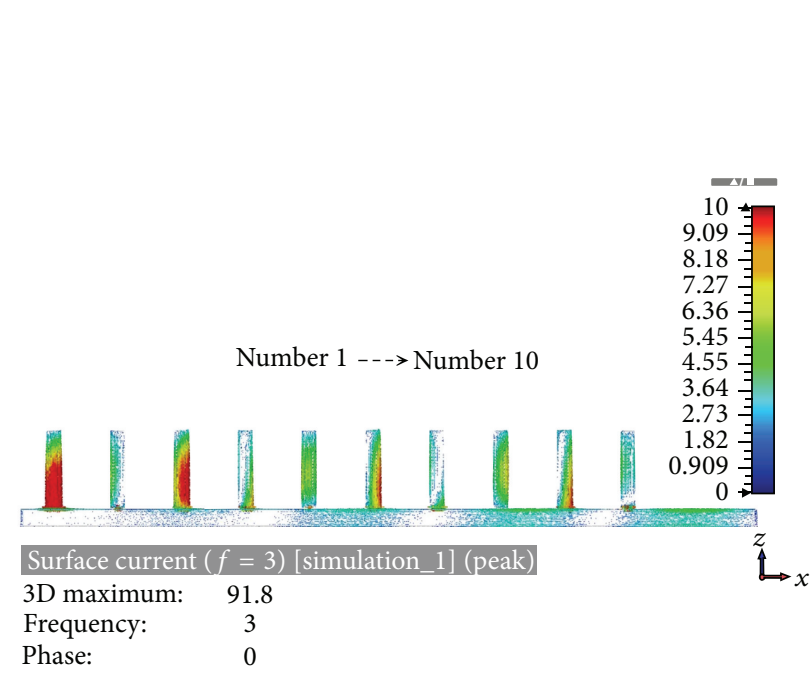

(a)

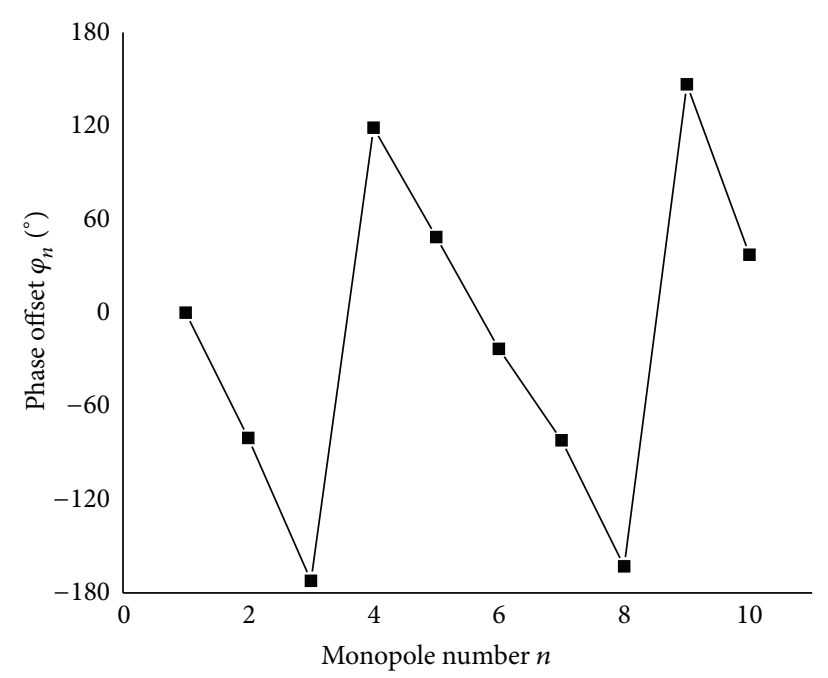

(b)

FIGURE 4: The current distribution on the 10-element monopole end-fire array with a finite ground $L_{a}=200 \mathrm{~mm}, L_{g}=30 \mathrm{~mm}$, and $W=$ $60 \mathrm{~mm}$ at $f=3 \mathrm{GHz}$ for a certain phase step $\Delta \varphi_{0}$ : (a) current vector on the surface, (b) actual current phase offsets of elements.

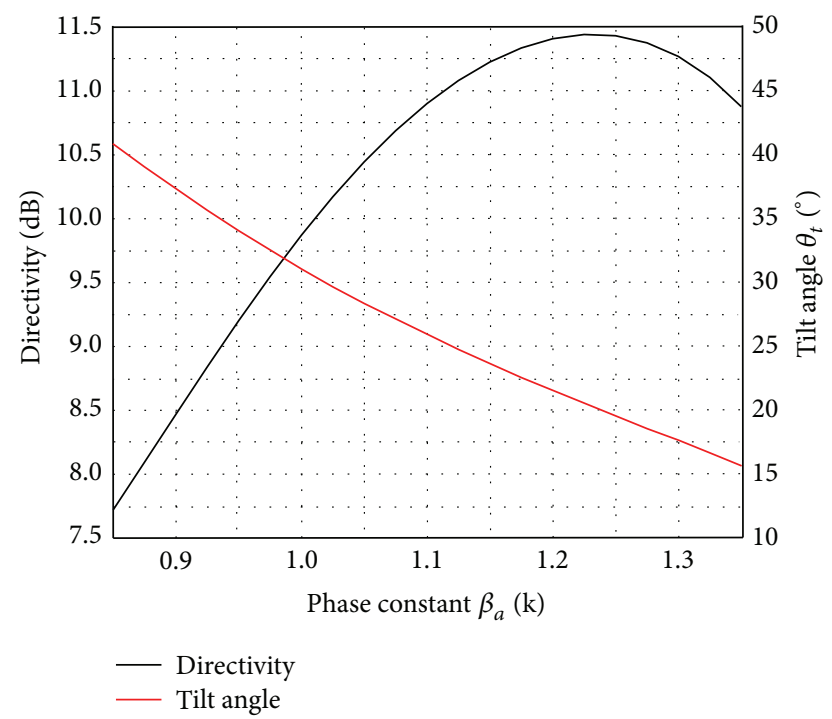

Figure 5: The relationships between $\beta_{a}$ and directivity, $\beta_{a}$ and tilt angle for 10-element monopole end-fire array with a finite ground $L_{a}=200 \mathrm{~mm}, L_{g}=30 \mathrm{~mm}$, and $W=60 \mathrm{~mm}$ at $f=3 \mathrm{GHz}$.

3.3. Discussion on the Significance of $\beta_{a}$ in Estimating. Based on the phenomenon shown above, we will explain the significance of wavenumber $\beta_{a}$ in the beam tilting estimation of monopole end-fire array.

Setting $L_{a}=2 \lambda$, the $\theta_{t}-L_{g}$ curves when $\beta_{a}=k, 1.1 k, 1.2 k$ and $1.3 k$ are, respectively, shown in Figure 8 compared with the curve of Walter's. The distinction between the condition $\beta_{a}=k$ and Walter's estimation, caused by the different vector current elements $\left(J_{z}, J_{x}\right)$ and $\left(K_{y}, J_{x}\right)[6]$, is not quite remarkable. However, as $\beta_{a}$ increases, the distinction is getting larger and $\theta_{t}$ with the same $L_{g}$ is getting lower. It is suggested that $\theta_{t}-L_{g}$ relationship is sensitive to $\beta_{a}$, and an
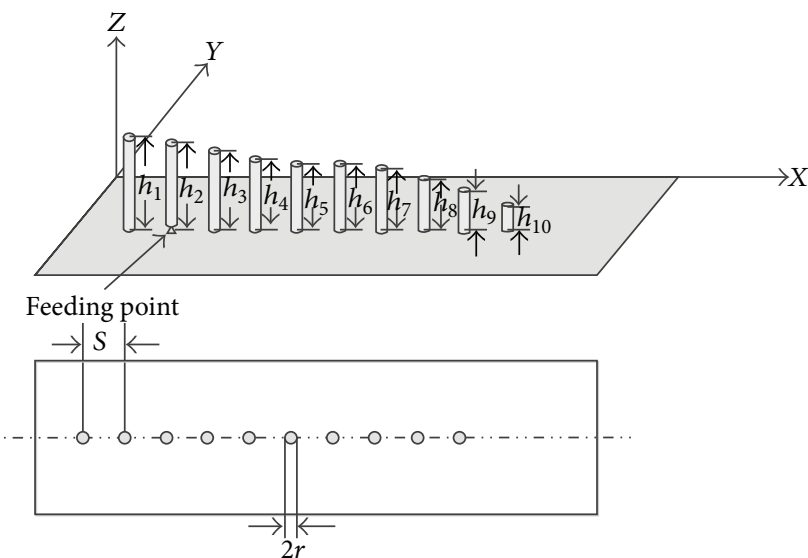

FIgURE 6: Geometry for the 10-element monopole Yagi-Uda antenna.

appropriate high value of $\beta_{a}$ can result in a low beam tilt angle in the range where ground plane is short.

By changing the array's feeding situation or structure parameters, the phase constant of monopole end-fire array can be adjusted in a wide range. Therefore, the evaluation of $\beta_{a}$ will determine the validity and accuracy of estimation.

\section{Experiment for 10-Element Monopole Yagi-Uda Antenna}

According to the analysis in Section 3, for a 10-element monopole end-fire array, when the total length of finite ground plane is about $L=L_{a}+L_{g}=2.3 \lambda$, the beam tilt angle is about $27^{\circ}$ when $\beta_{a}=k$. For the same length, when $\beta_{a}$ is satisfying Hansen-Woodyard condition the tilt angle can be 

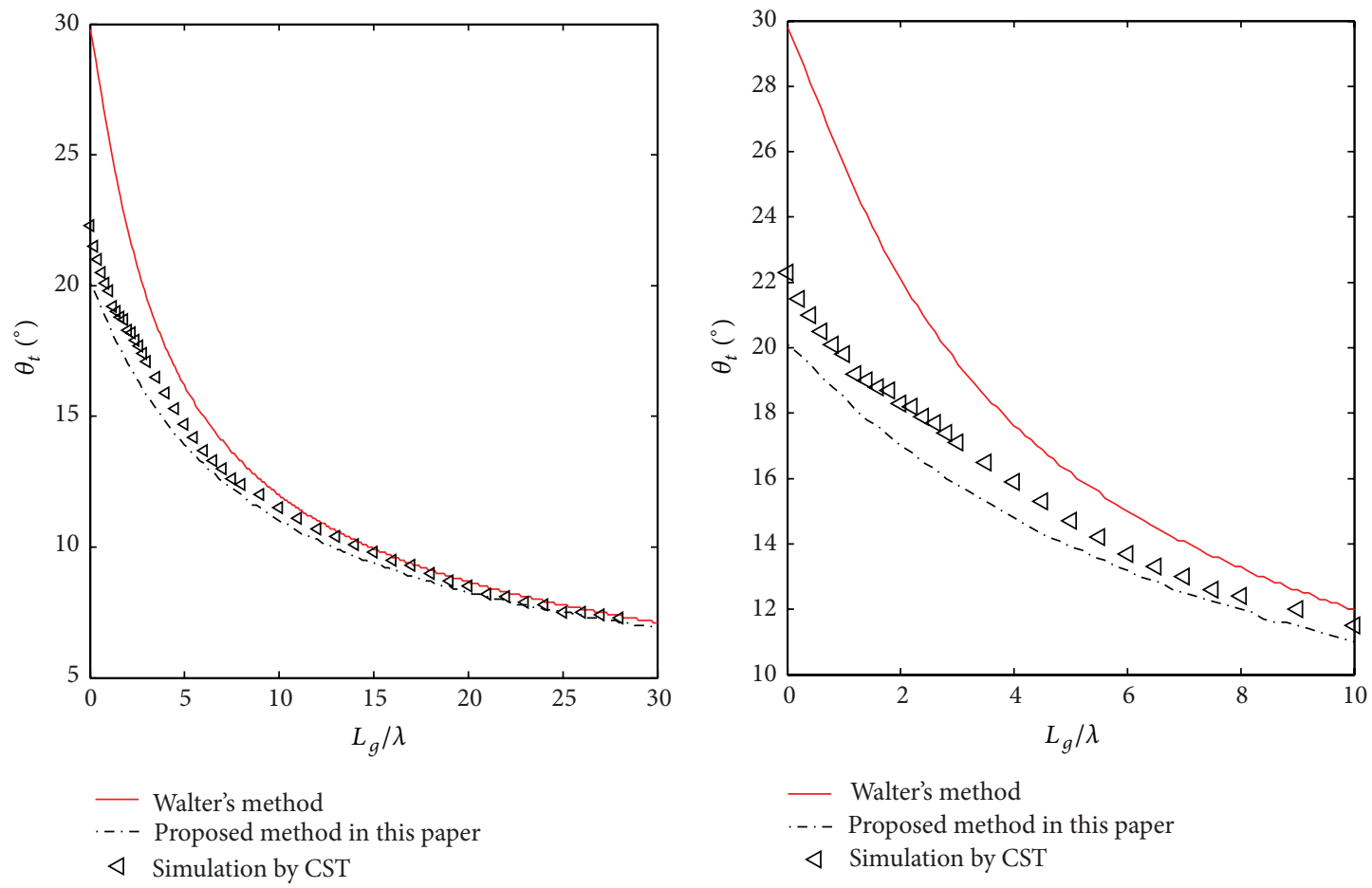

FIGURE 7: Beam tilt angles of the 10-element antenna when $L_{a}=200 \mathrm{~mm}$, got by the proposed method in this paper, Walter's method, and CST simulating.
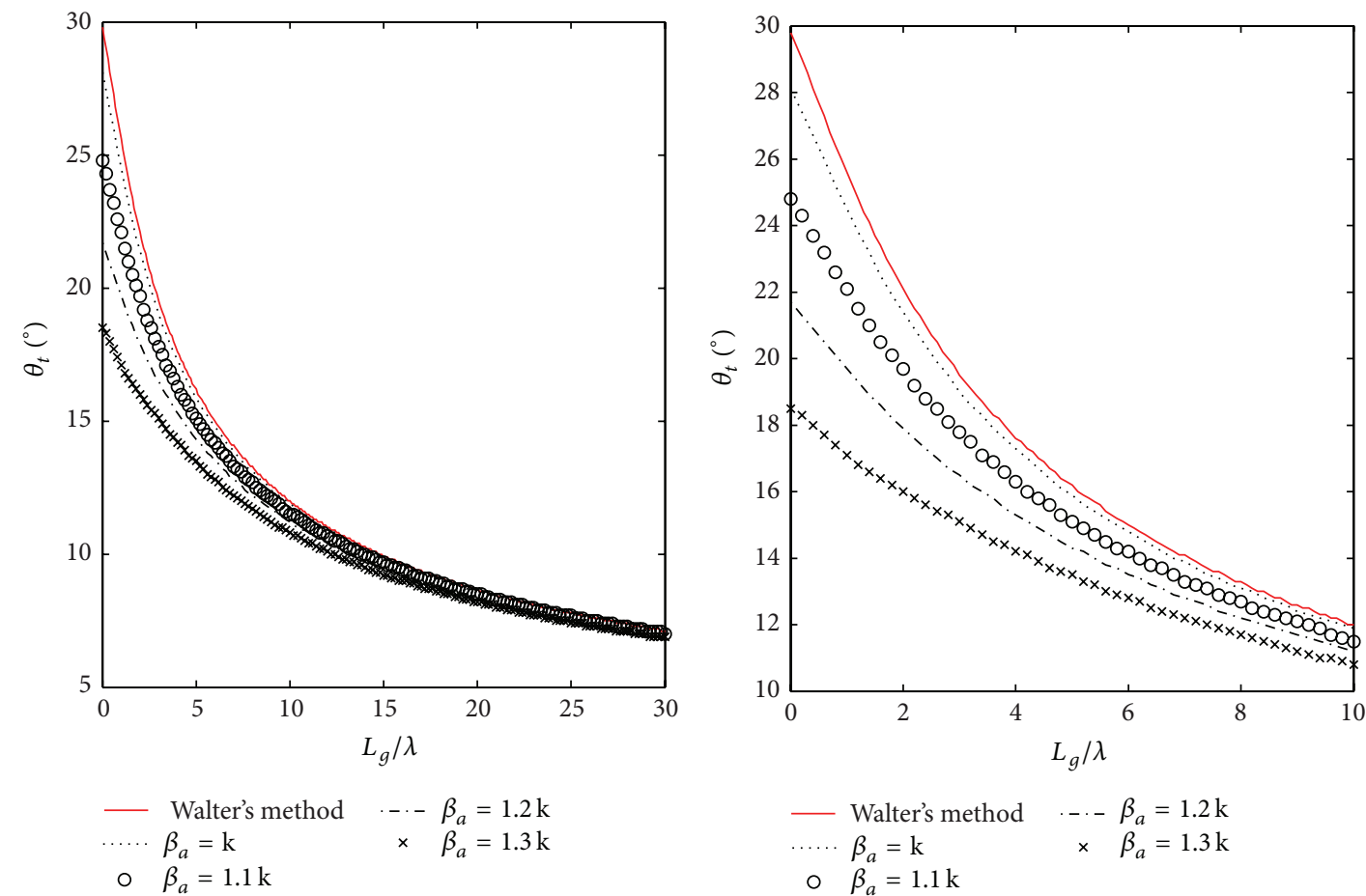

FIGURE 8: Estimated beam tilt angles of the 10-element monopole end-fire antenna when $L_{a}=2 \lambda, \beta_{a}=k, 1.1 k, 1.2 k$ and $1.3 k$ and Walter's Method. 


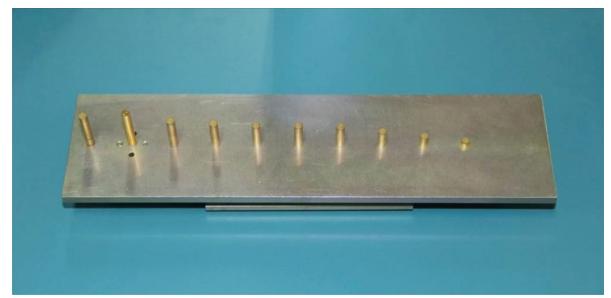

Figure 9: Photo for the 10-element monopole Yagi-Uda antenna mounted on a finite ground plane with size $L=230 \mathrm{~mm}, W=60 \mathrm{~mm}$.
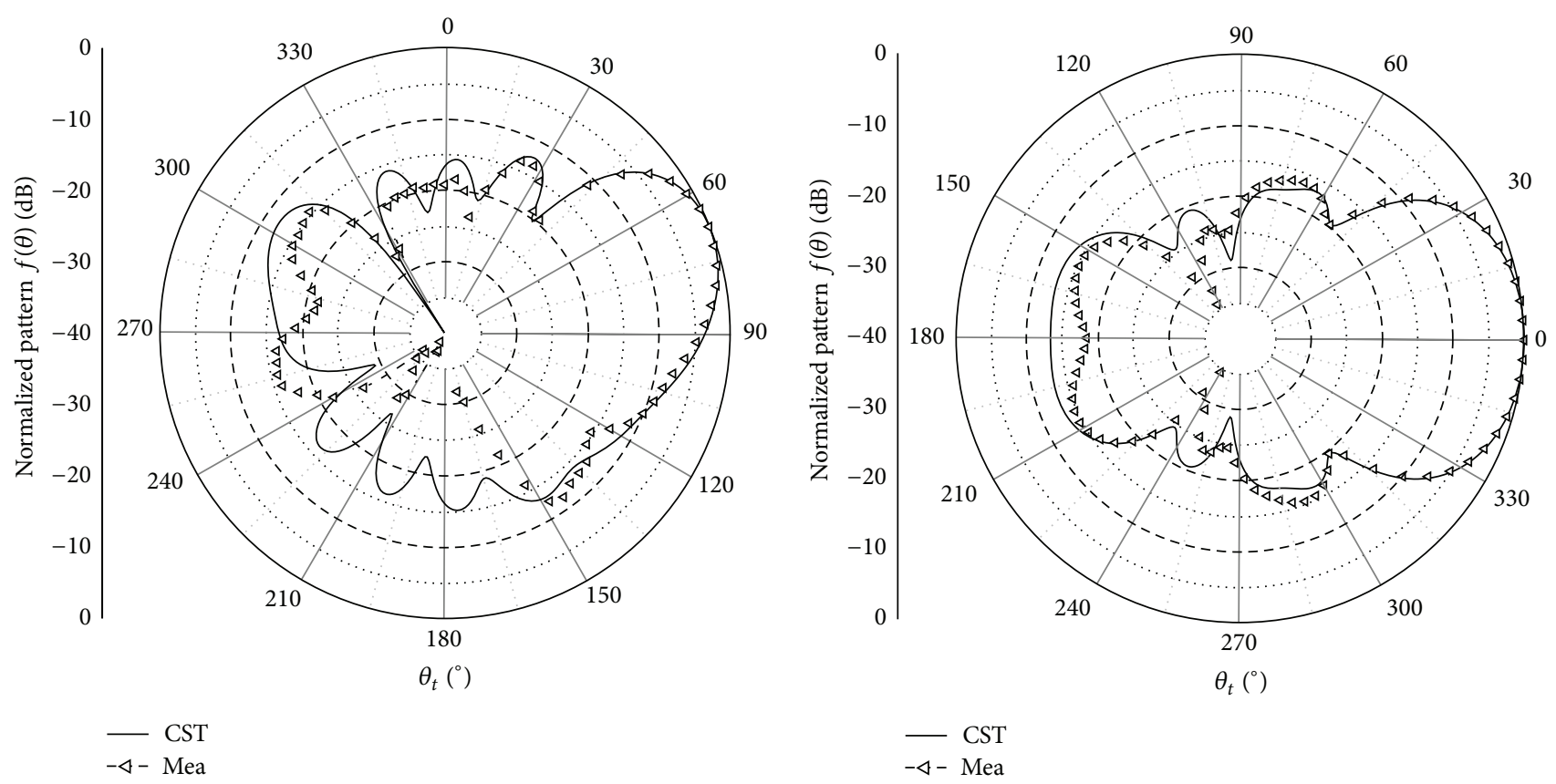

FIGURE 10: Measured radiation pattern for the 10-element monopole Yagi-Uda antenna shown in Figure 9.

lowered to about $20^{\circ}$, which needs a ground plane whose total length $L=L_{a}+L_{g} \geq 4.5 \lambda$ when $\beta_{a}=k$.

The 10-element monopole Yagi-Uda antenna, which satisfies this condition as mentioned in Section 3, is fabricated and measured to prove this deduction. Parameters for the 10 monopole array are totally the same as those given in Table 1. The size of finite ground plane is $L=230 \mathrm{~mm}, W=60 \mathrm{~mm}$. The photo of the fabricated antenna is shown in Figure 9.

Simulation and measured results for the elevation $(X Z$ plane) and azimuth ( $X Y$-plane) radiation patterns at $3 \mathrm{GHz}$ are shown in Figure 10. This antenna achieves about $11.56 \mathrm{~dB}$ gain in practice and the main beam point at tilt angle of about $21^{\circ}$ from end-fire, which is very close to the estimated tilt angle $20^{\circ}$ at the beginning of this section. This experimental result demonstrates not only the accuracy of the estimation method proposed in this paper, but also the conclusions deduced at the end of Section 3: the beam tilt angle is sensitive to $\beta_{a}$, and an appropriate $\beta_{a}$ can lower the angle with a short ground plane.

\section{Conclusion}

A modified method for the beam tilt-angle estimation of endfire antenna mounted on finite ground plane is proposed and studied. This method focuses on analyzing the case of monopole end-fire array. The monopole array and ground plane are simplified into two line sources of transverse and longitudinal current source, respectively. Superposition results of two line sources suggest the beam tilt angle is a function about the length of ground plane in front of array $L_{g}$, the length of monopole array $L_{a}$, and the phase constant $\beta_{a}$. Evaluating the value of $\beta_{a}$ correctly is demonstrated to be the key point of this estimation method. A low beam tilt angle can be achieved by a monopole array satisfying HansonWoodyard condition with only a short-length ground plane. A 10-element monopole Yagi-Uda antenna is designed and measured for the analysis. It produced a tilt angle about $21^{\circ}$, which is close to the estimation value, with a total ground size $L=230 \mathrm{~mm}, W=60 \mathrm{~mm}$. 


\section{Conflict of Interests}

The authors declare that there is no conflict of interests regarding the publication of this paper.

\section{References}

[1] W. Cao, B. Zhang, A. Liu, T. Yu, D. Guo, and Y. Wei, "Gain enhancement for broadband periodic endfire antenna by using split-ring resonator structures," IEEE Transactions on Antennas and Propagation, vol. 60, no. 7, pp. 3513-3516, 2012.

[2] X. Ding and B. Z. Wang, "A novel wideband antenna with reconfigurable broadside and endfire patterns," IEEE Antennas and Wireless Propagation Letters, vol. 12, pp. 995-998, 2013.

[3] Y. H. Sun, G. J. Wen, H. Y. Jin, P. Wang, and Y. J. Huang, "Gain enhancement for wide bandwidth endfire antenna with I-shaped resonator (ISR) structures," Electronics Letters, vol. 49, no. 12, pp. 736-737, 2013.

[4] J. Liu and Q. Xue, "Microstrip magnetic dipole yagi array antenna with endfire radiation and vertical polarization," IEEE Transactions on Antennas and Propagation, vol. 61, no. 3, pp. 1140-1147, 2013.

[5] R. Elliott, "On the theory of corrugated plane surfaces," IRE Transactions on Antennas and Propagation, vol. 2, no. 2, pp. 7181, 1954.

[6] C. H. Walter, Traveling Wave Antennas, McGraw-Hill, New York, NY, USA, 1965.

[7] H. Nakano, Y. Ogino, and J. Yamauchi, "Bent two-leaf antenna radiating a tilted, linearly polarized, wide beam," IEEE Transactions on Antennas and Propagation, vol. 58, no. 11, pp. 3721-3725, 2010. 

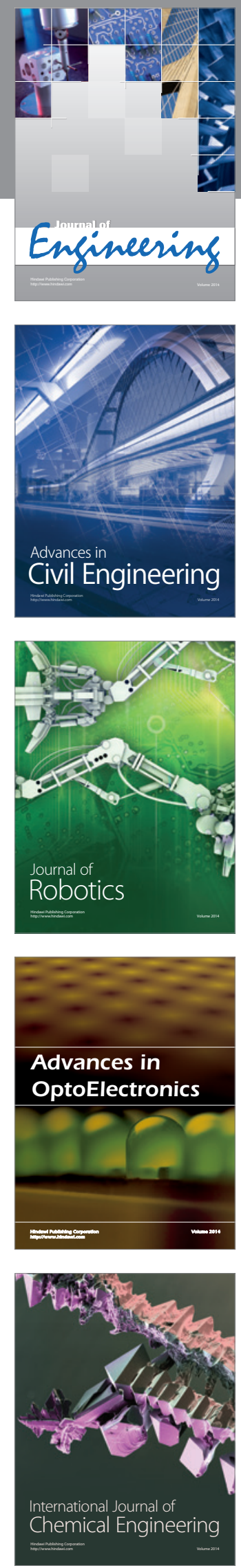

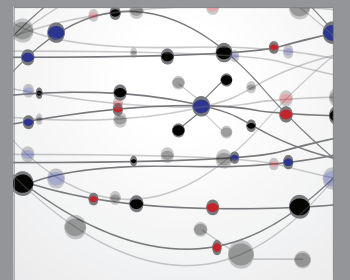

The Scientific World Journal
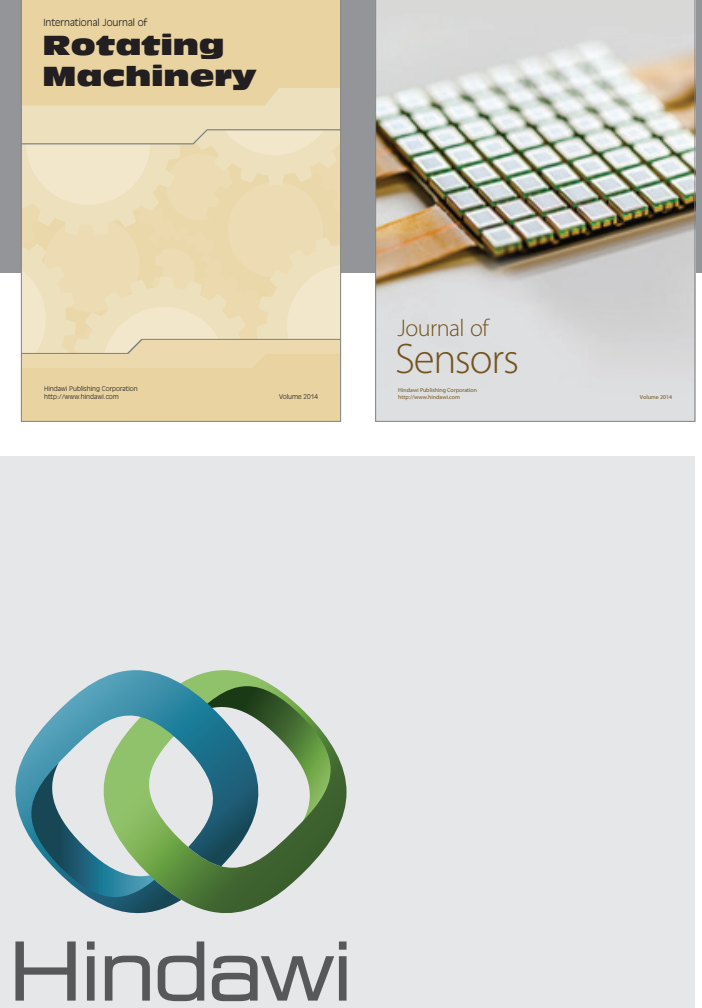

Submit your manuscripts at http://www.hindawi.com
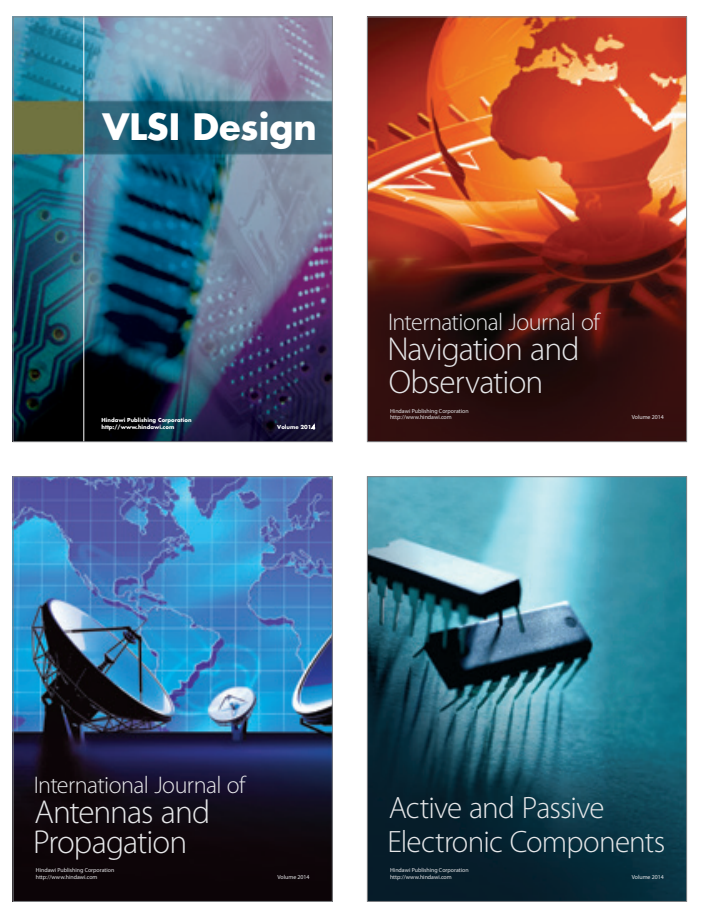
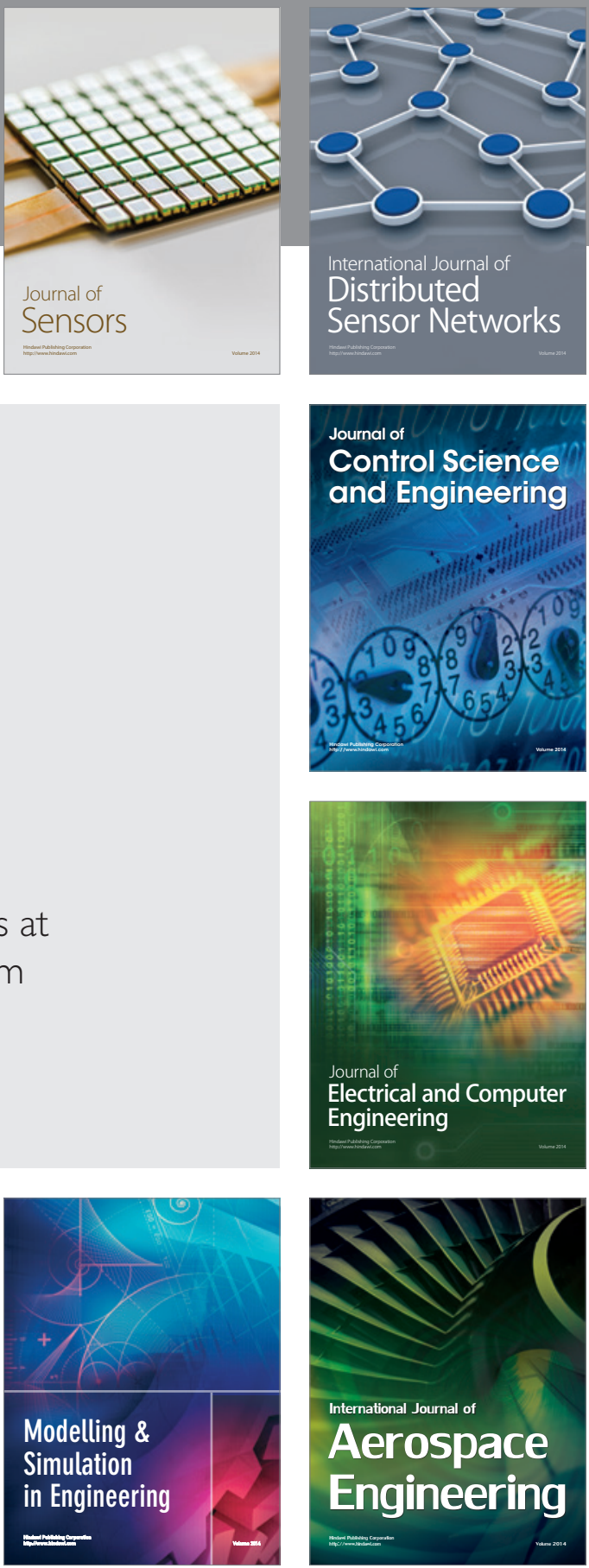

Journal of

Control Science

and Engineering
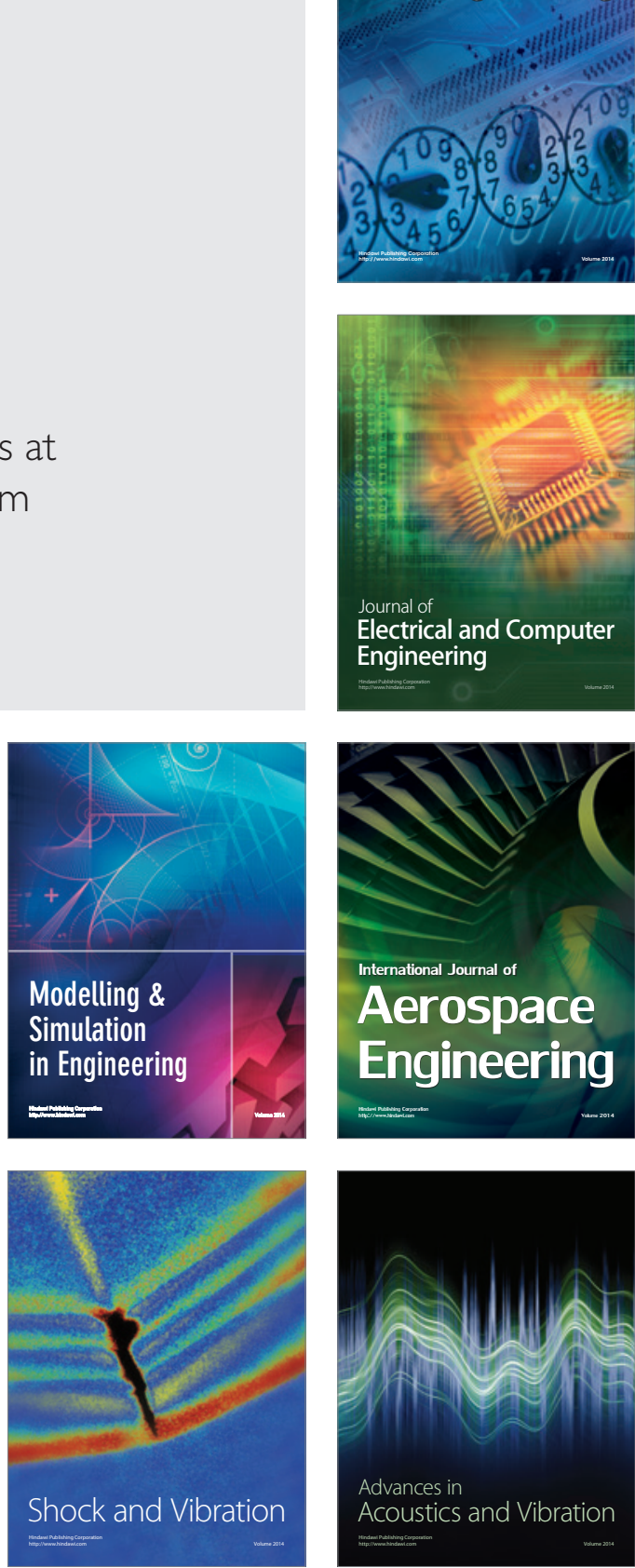